\title{
Development of an Autonomous Quadrotor with a Robust Real- time Control: A Review on Modeling, Estimation, Control, and Hardware Aspects
}

This paper was downloaded from TechRxiv (https://www.techrxiv.org).

\section{LICENSE}

CC BY-NC-SA 4.0

SUBMISSION DATE / POSTED DATE

$02-12-2021 / 11-12-2021$

\section{CITATION}

Goswami, Mayank; Kumar, Ankur; Chavan, Pradnesh (2021): Development of an Autonomous Quadrotor with a Robust Real-time Control: A Review on Modeling, Estimation, Control, and Hardware Aspects. TechRxiv. Preprint. https://doi.org/10.36227/techrxiv.17111066.v1

$\mathrm{DOI}$ 


\title{
Development of an Autonomous Quadrotor with a Robust Real-time Control: A Review on Modeling, Estimation, Control, and Hardware Aspects
}

\author{
Pradnesh Chavan, Ankur Kumar, Mayank Goswami \\ Department of Physics, Indian Institute of Technology Roorkee
}

\begin{abstract}
The following report is a comprehensive discussion on the development of a resilient autonomous quadrotor equipped with a robust control mechanism for optimal performance. An introduction to quadrotor modeling and flight dynamics is provided first. The autopilot control and state estimation methods are then described from both software and hardware viewpoints. A review of PX4 autopilot control architecture is provided to comprehend a complete control system integration. It is followed by a survey of commonly used sensors, micro-controllers, actuators, and other hardware peripherals used in academic and commercial grade quadrotors, along with their architectural overview. Next, a brief discussion on the software components essential for a real-time implementation of the developed control system on the hardware is done. Finally, concluding remarks are made on each stage of quadcopter development, and potential research problems are forecasted.
\end{abstract}

Keywords-Quadrotor, Flight Control, Rigid-body Dynamics, Autopilot Systems, Sensors, Embedded Systems.

\section{INTRODUCTION}

Aerial robotics has been an active research topic for decades, but it has attracted the interest of several drone hobbyists, industry personnel, and researchers in recent years. These aerial vehicles, also colloquially known as drones or UAVs (Unmanned Aerial Vehicles), have sufficient payload carrying capacity and sophisticated control systems that enable their use for a plethora of applications in both indoor and outdoor environments. Innovative drone-based solutions are being adopted each day from the defense and military to the commercial sector due to the rapid advancements in battery, sensors, and microprocessor technology. Aerial photography and mapping, transportation, FPV drone racing, search and rescue missions are a few popular applications of these vehicles.

Depending on the type of airframe used, the aerial vehicles can be classified as multirotor, fixed-wing aircraft, or VTOL (Vertical Takeoff and Landing) crafts. Due to their portability and ease of operation, multirotor aircraft, especially quadrotors, i.e., vehicles with four individual rotors attached to a cross airframe, have become a standard experimental platform for academic research. With the availability of several open-source autopilot controllers such as PX4, Ardupilot, etc., and all-in-one integrated drone development platforms such as the PX4 Vision Autonomy Development Kit or the Holybro Kopis 2, researchers can focus on their respective domains without having to worry much about the underlying system specifications. Nevertheless, the cost of these fully assembled platforms and other limitations, such as limited processing capacity of the onboard processor, which often forces the use of off-board components for computing; slender payload carrying capacity, and restrictions on payload placements, especially in the case of micro and nano quadrotors such as the Bitcraze Crazyflie [1] pose a severe restriction on further research. This has resulted in an increase in the number of self-developed and manufactured drone models, making innovation either in the vehicle hardware or the autopilot flight control or the mechanical components of these drones.

A lot of existing literature, such as the PX4 documentation [2], serves as a great starting point for those who're new to the field of drones and automation. However, a majority of these resources only provide an architectural overview of the drone model without giving an intuitive idea about the intricate components used while developing such a system. At the same time, they do not include the reasoning behind their design choices, for e.g., why a sensor and MCU combination is suitable for performing a specific flight task.

The main objective of this report is to provide an overview of the different stages of development involved in making an autonomous quadrotor. Each section serves as a prelude, providing sufficient background information about the topic, followed by a brief review of the state-of-the-art technology used to implement that stage, an analysis of a few of these technologies based on existing benchmarks, and a survey of potential research areas and directions in that domain. Section II summarizes the rigid-body dynamics and aerodynamics of the airframe and rotors used for vehicle modeling. An analysis of the drone's mechanical components and its overall effect on the vehicle performance is provided. Section III introduces the concept of state estimation, explaining how the different lowlevel sensors such as accelerometers and gyroscopes can be used to estimate the drone's parameters which could be used for its relative positioning. It introduces the concepts of Kalman Filtering and how it improves the positional accuracy using sensor fusion techniques. Section IV presents the control problem for autonomous drones and describes how a variety of controllers work in synchronization using feedback loops to enable these vehicles to perform autonomously, i.e., the way we interpret them. Section V provides an architectural overview of the different hardware components commonly used in autonomous UAVs along with their functioning and a comparison of a few of them by comparing their datasheets and experimental results from a few papers. Section VI focuses on the microcontroller units used for onboard as well as off-board processing. The timing and update 
requirements of the autopilot controllers at a hardware level, common middleware suites, use of a companion computer, and methods of integrating all these components are discussed. Another main objective of our work is to develop our own quadrotor model from scratch with a bias towards nano-scale UAVs similar to the CrazyFlie but having onboard processing capability. For this, a briefcase study of ETH Zurich's PULP-GAP8 embedded processor is provided.

\section{MODELING AND FLIGHT DYNAMICS}

A quadrotor consists of four rotors attached to a rigid crossframe. These four rotors are actuated using the attached motors to either enable translational (along the three mentioned axes, namely: $\mathrm{X}, \mathrm{Y}, \mathrm{Z}$ ) or rotational (along the three rotational axes, namely: Roll, Pitch, Yaw) motion. The two opposite rotors along the body frame have the same spin. Depending upon the direction of net torques and thrust due to each rotor, the drone moves along these $6 \mathrm{DOF}$ (Degrees of Freedom), and this is governed using the concepts flight dynamics.

\section{A. Rigid-Body Dynamics and Aerodynamic Effects}

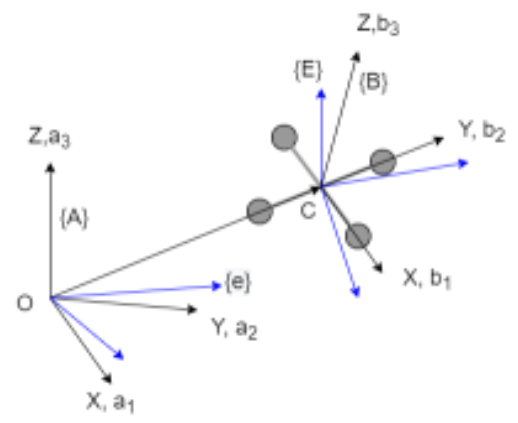

Fig. 1. Quadrotor Model

Consider the following model for a quadrotor located in 3dimensional space as depicted in Figure 1. Following the righthand convention, let $\{\mathrm{A}\}$ denote the inertial frame and $\{\mathrm{B}\}$ denote the body-fixed frame for the drone. The corresponding unit vectors in both frames are $\left\{\vec{a}_{1}, \vec{a}_{2}, \vec{a}_{3}\right\}$ and $\left\{\vec{b}_{1}, \vec{b}_{2}, \vec{b}_{3}\right\}$ respectively. By rotating the drone through a roll angle, $\varphi$; pitch angle, $\theta$; and yaw angle, $\psi$; we can obtain the rotation matrix between the two coordinate frames $\{\mathrm{A}\}$ and $\{\mathrm{B}\}$ as follows:

$$
R=\left(\begin{array}{ccc}
c \psi c \theta-s \phi s \psi s \theta & -c \phi s \psi & c \psi c \theta+c \theta s \phi s \psi \\
c \theta s \psi+c \psi s \phi s \theta & c \phi c \psi & s \psi s \theta-c \psi c \theta s \phi \\
-c \phi s \theta & s \phi & c \phi c \theta
\end{array}\right)
$$

where $\mathrm{c}$ and s stand for cosine and sine functions, respectively. This rotation matrix provides the orientation of the body-fixed frame, where $\operatorname{R\in SO}(3)$ is in the special orthogonal group. If $\xi$ denotes position, $v$ and $\Omega$ denote the linear and angular velocities of frame $\{B\}$ w.r.t $\{A\}$; m denote the mass of the rigid body and $I \in \mathbb{R}^{3 \times 3}$ denote the inertia matrix in frame $\{B\}$. Then the equations of motion can be written as:

$$
\begin{gathered}
\dot{\xi}=v \\
m \dot{v}=m g+R F \\
\dot{R}=R \Omega \\
I \dot{\Omega}=-\Omega \times I \Omega+\tau
\end{gathered}
$$

where $F$ and $\tau$ are the non-conservative forces and moments applied to the quadrotor due to the aerodynamic effect of the rotors.

For a hovering rotor at steady-state, the thrust generated by the rotor can be obtained using the momentum theory as:

$$
T i=C_{T} \rho A r^{2} \omega^{2}
$$

where A: Rotor disk area, r: radius, $\rho$ : density and $C_{T}$ is a constant called thrust co-efficient dependent on the rotor geometry whose value is determined experimentally.

Similarly, the reaction torque generated at each rotor is given by:

$$
\mathcal{Q}_{i}=C_{Q} \omega^{2}
$$

where $C_{Q}$ is also determined by static thrust tests.

The total thrust $(T \Sigma)$ while hovering is simply the sum of thrusts from the individual rotor and contributes to the aerodynamic force $F$ present in the equation of motion. Similarly, the net moment $(\tau)$ is generated due to all these aerodynamic forces (i.e., the combined effect of produced torque and air resistance) and is represented as:

$$
\begin{gathered}
\tau=\left(\tau_{1}, \tau_{2}, \tau_{3}\right) \\
\tau_{1}=C_{T} \sum_{i=1}^{4} d_{i} \sin \left(\phi_{i}\right) \omega_{i}^{2} \\
\tau_{2}=-C_{T} \sum_{i=1}^{4} d_{i} \cos \left(\phi_{i}\right) \omega_{i}^{2} \\
\tau_{3}=C_{Q} \sum_{i=1}^{4} \sigma_{\mathrm{i}} \omega_{i}^{2}
\end{gathered}
$$

All these equations can be written in a combined matrix form as:

$$
\left(\begin{array}{c}
T_{\Sigma} \\
\tau_{1} \\
\tau_{2} \\
\tau_{3}
\end{array}\right)=\left(\begin{array}{cccc}
C_{T} & C_{T} & C_{T} & C_{T} \\
0 & d C_{T} & 0 & -d C_{T} \\
-d C_{T} & 0 & d C_{T} & 0 \\
-C_{Q} & C_{Q} & -C_{Q} & C_{Q}
\end{array}\right)\left(\begin{array}{c}
\omega_{1}^{2} \\
\omega_{2}^{2} \\
\omega_{3}^{2} \\
\omega_{4}^{2}
\end{array}\right)
$$

This expression easily abstracts the underlying equations since we can easily determine the speed of the rotors provided the required thrust and moment for each rotor is known. For steady-state hovering, $T \Sigma$ should be equal to $\mathrm{mg}$ and net moment should be zero. This can be achieved by feeding in suitable values for motor speed using the above matrix expression.

The type of material used for designing the rotors also plays a crucial role in modeling the UAV. Most of the vehicles contain non-rigid, plastic rotors which can bend and deform slightly due to the aerodynamic and inertial forces. A stiff, rigid rotor could transmit these forces to the body-frame and lead to structural damage. Taking this into account, standard sized quadrotors should preferably have rotors built with material having less rigidity. 
Another important observation in the case of quadrotors in steady-state hovering is a slight horizontal and vertical displacement about the set position. Similar to the phenomena of lift and drag in airplanes generated due to a difference in the airspeed at the top and bottom of the wings, quadrotor blades have different absolute tip velocities. Typically the advancing blade has higher tip velocity and generates more lift than the retreating blade. This effect, also known as 'Rotor Flapping', is usually countered at steady-state hovering at high angular momentum rates, and the UAV nearly stays in equilibrium. However, in the case of small vehicles, due to their relatively rigid blades, this effect always ensures a presence of a noticeable horizontal drag. A detailed mathematical modeling of this effect can be found in [3]. This is another reason why control of micro and nano UAVs is a topic of research in today's time.

Finally, it's also worth mentioning the effect of changing the UAV dimensions on its performance. Mach Scaling, a commonly used approach for compressible flows, proves that angular rate increases with a reduction in drone dimensions, whereas the linear acceleration remains at worst unaffected. This is the reason why smaller UAVs are more robust and acrobatic compared to larger ones.

\section{STATE ESTIMATION}

Having described the equations governing quadrotor dynamics, we now consider the important state variables before defining the control problem for the drone. The 12 key state variables required for optimal $\vec{c}$ ontrol are:

$$
\begin{array}{r}
\text { Position }:\{x, y, z\} \\
\text { Velocity }:\{\dot{x}, \dot{y}, \dot{z}\} \\
\text { Attitude }:\{\phi, \theta, \psi\} \\
\text { Angular Velocity }:\{\dot{\phi}, \dot{\theta}, \dot{\psi}\}
\end{array}
$$

The variety of sensors onboard these vehicles helps in providing a rough estimate for these states. More frequently, filters are used to process the inherent noise present in the readings, and sensor fusion techniques are utilized for precise state estimation.

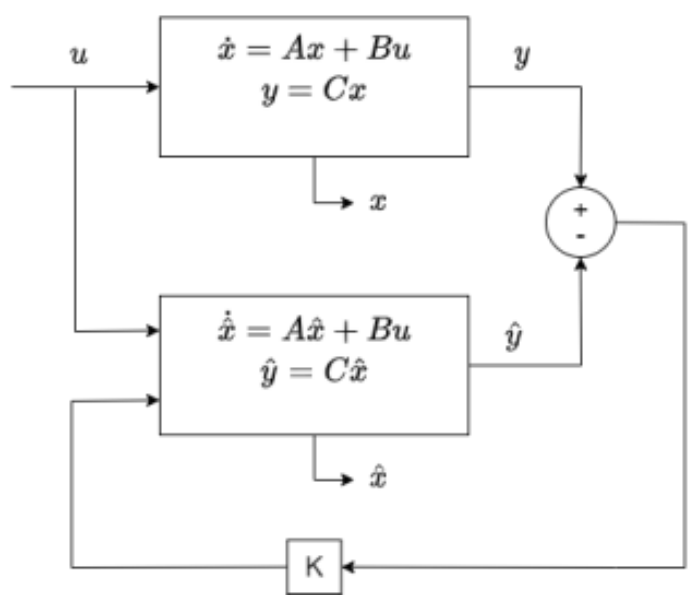

Fig. 2. State Observer
The state estimation problem can be formulated as follows: For a given system shown in Figure 2 whose mathematical model is known, let $u$ and $y$ denote the input and output, respectively, and $\mathrm{x}$ is the state to be estimated since it cannot be measured directly. Let $\hat{x}$ and $\hat{y}$ denote the estimated values using a mathematical model. The goal is to minimize the difference between $\hat{x}$ and $x$ by minimizing the difference between $\hat{y}$ and $y$, i.e., try to make our mathematical model as robust as possible. Defining a control loop with gain $\mathrm{K}$ around the state observer, we try to eliminate the error between the estimated and measured output. Error dynamics for this feedback system can be given as follows:

$$
\begin{aligned}
& \dot{x}-\dot{\hat{x}}=A x-A \hat{x}+B u-B u+K(y-\hat{y}) \\
& y-\hat{y}=C(x-\hat{x})=C e_{o} \\
& \dot{e}_{o}=(A-K C) e_{o}
\end{aligned}
$$

Solution to the above equation is an exponential function.

If $(A-K C)$ tends to 0 , then the error term will tend to 0 and $x$ and $\hat{x}$ will converge. Also, it's worth mentioning that if the system modeling is not precise, then matrix A won't be accurate enough for fast convergence of the error. By tuning the gain $\mathrm{K}$ of the feedback loop, it is viable to control the rate of convergence which is precisely done by techniques such as Kalman filters in the presence of process and measurement noise.

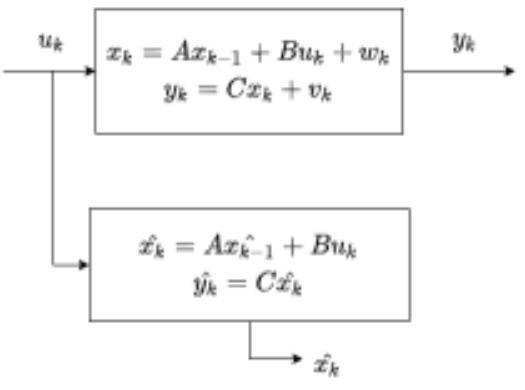

Fig. 3. Optimal State Estimator

Consider another model as shown in Figure 3. The difference between the present and the previous model is the presence of process and measurement noise in the underlying equations i.e. $w_{k}$ and $v_{k}$ respectively. This resembles the sensor readings of onboard drone sensors, which are also susceptible to noise. Such random variables don't essentially follow a pattern, but it is still possible to predict their average properties using probability theory. For instance, $v$ and $w$ can be assumed to be a Gaussian distribution of variance $R$ and $Q$, respectively. The mathematical model for this system won't likely resemble these noisy random variables; thus, the estimate $\hat{x}_{k}$ won't be accurate since we're estimating $x$ which contains inherent noise. Kalman filter combines this measurement and prediction to find the optimal estimate of the output by multiplying the two probability density functions together, resulting in a Gaussian distribution whose mean is the optimal estimate of the output.

Having covered the basics of state estimation, we now study their basic implementation using UAV sensors. 


\section{A. Attitude Estimation}

Inertial Measurement Units (IMUs) are present in almost all quadrotors and are typically comprised of a 3-axis gyroscope, accelerometer, and magnetometer, respectively. The following equations summarize the measurement values provided by each of the individual components of an IMU:

$$
\begin{gathered}
\Omega_{I}=\Omega+b_{\Omega}+\eta \\
a_{I}=R(\dot{v}-\mathrm{g} \vec{z})+b_{a}+\eta_{a} \\
m_{I}=R m^{A}+B_{m}+\eta_{b}
\end{gathered}
$$

where gyro reading gives the angular velocity of $\{\mathrm{B}\}$ w.r.t $\{\mathrm{A}\}$; accelerometer measures the instantaneous linear acceleration of $\{\mathrm{B}\}$ to external forces; magnetometer `reading gives the ambient magnetic field in the presence of bias $b_{i}$ and measurement noise $n_{i}$ respectively for each sensor. Using mathematical models assuming a suitable initial bias and measurement noise, the accelerometers, with appropriate low-pass filters and magnetometers, can be used to provide attitude data, whereas the gyroscope can be used to measure the angular velocity. Similarly, using the attitude equations described in Section II and appropriate state observers, for e.g., non-linear complementary filters, it is possible to fuse the accelerometer, gyro, and magnetometer data. This, unlike the Kalman filter, won't be able to track the senor bias frequently, but one important advantage is that the gains can be adjusted in realtime by ensuring the bias is small. For e.g., this filter would set the accelerometer gain to 0 while doing fast maneuvers and would consider the accelerometer reading while the drone is in a stable hovering state.

\section{B. Velocity Estimation}

Once the attitude estimation is done, the kinematics of rotor flapping can be used to generate a state observer and calculate a horizontal component of acceleration assuming a constant height. Integrating this estimate overtime at a suitable frequency helps in achieving a precise estimate of the horizontal velocity.

\section{Position Estimation}

The position of a quadrotor in 3D space can be expressed either in an absolute or a relative way. The following table sums up the traditional approaches for position estimation:

TABLE I

TECHNIQUES USED FOR POSITION ESTIMATION

\begin{tabular}{cc}
\hline Quadrotor's & Sensor and approach used \\
Coordinate & GPS/VICON motion capture \\
\hline Absolute X and & \\
Y & Laser ranger finders/ RGBD cameras \\
Relative X and & \\
Y & Barometer/GPS/VICON motion capture \\
Absolute height & system/Vision-based SLAM method \\
Relative height & Acoustic/Laser/Infrared sensors \\
\hline
\end{tabular}

\section{FLIGHT CONTROL}

The control problem of actuating the quadrotor system with 6 DOF having only four input variables: total thrust and moments along each individual axis involve developing algorithms for trajectory planning and state variable control.

Consider a system with a feedback loop where the actuating signal is driven through the plant to generate a controlled variable. And based on a pre-defined set-point or a commanded variable, the goal is to minimize the error between the commanded and the controlled variable, i.e., the set-point and the output of the system. A controller is used to take in this error term and convert it into suitable actuator commands so that over time the error term is driven to zero.

A PID (Proportional-Integral-Derivative Controller) is often implemented as a controller to influence the UAV's position, attitude, and velocities. The following is an expression for the output of a PID controller in a closed-loop system in the time domain:

$$
u(t)=K_{p} e(t)+K_{i} \int e(t) d t+k p \frac{d e}{d t}
$$

Each individual component of the PID acts on the error variable, and the magnitude of its effect is calculated based on the value of the constants $\mathrm{Kp}, \mathrm{Kd}$, and $\mathrm{Ki}$ whose values are evaluated after a proper tuning of the system. Laplace transform of equation $\mathrm{x}$ gives the transfer function of the controller:

$$
\frac{K_{d} s^{2}+K_{P} s+k_{i}}{s}
$$

The values of the coefficients have a significant impact on the performance of the closed-loop system, and in the case of quadrotors, these are often used in combination to get the desired effect. The proportional component drastically affects the reaction time; however, at the same time, affecting the system's stability by causing an overshoot. To avoid this, a derivative controller is often used as it introduces damping into the system; however, neither of them affects the steady-state error. The integrator, on the other hand, increases the control signal further to compensate for the steady-state error however introduces oscillations into the system. The following table summarizes the effect of tuning constants on the behavior of the system.

TABLE II

EFFECT OF PID COEFFICIENTS

\begin{tabular}{ccccl}
\hline $\begin{array}{c}\text { Co- } \\
\text { efficient }\end{array}$ & Rise & Over- & Settling & Steady State \\
Time & shoot & Time & Error \\
$\mathrm{Kd}$ & Decrease & Increase & No change & Decrease \\
& No & Decrease & Decrease & No change \\
$\mathrm{Ki}$ & change & & & \\
& Decrease & Increase & Increase & Decrease \\
\hline
\end{tabular}

In the case of quadrotors, a high-quality control of the motor speeds is crucial for robust closed-loop feedback control. A typical controller architecture based on cascaded loops as implemented in the common autopilots such as PX4, Ardupilot, etc., is as shown in Figure 4. The angle and angular rate controllers measured in the body frame constitute the innermost motor control loop, updating 


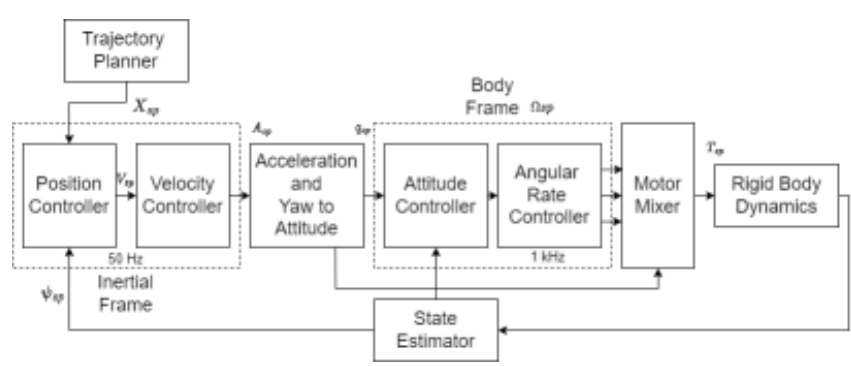

Fig. 4. Cascaded Controller Architecture

the values at comparatively high frequencies of $250 \mathrm{~Hz}-1 \mathrm{kHz}$. The position and velocity controllers form the outer control loop and take into consideration the inertial frame values of position and velocity estimates obtained from the state estimator block and operate at a comparatively low frequency of $50 \mathrm{~Hz}$. Each of these controllers makes use of PID control at the lowest level. Appropriate low-pass filters are applied to the derivatives block and notch filters to mitigate the noise. The inner controller produces actuator commands based on the set-points provided by the outer controller. The mixer receives these commands and mixes them with the thrust to generate the final motor commands.

Each commercially available flight controller has different update frequencies. On every update of $400 \mathrm{~Hz}$ on Pixhawk, 100 $\mathrm{Hz}$ on APM2.x, the following process is executed:

- Interpreting the pilot command provided by the autopilot (in case of autonomous UAVs) or operator (in case of manual UAVs) and setting the target roll, pitch, yaw rates

- Calculating the attitude error and converting to roll, pitch, yaw rotation rates

- Calculating rotation rate error and converting to high-level motor requests

- Converting the high-level motor requests to individual motor outputs

- Final scaling of outputs

- Sending final Pulse-width modulated (PWM) signals to the Electronic Speed Controllers (ESCs)

Finally, it is worth mentioning a few important things about PID tuning. Typically before developing a control architecture, system identification is made, i.e., understanding the system in consideration and analyzing its requirements. Parameters such as the linearity of the system, identifying how fast the system response should be either based on frequency characteristics (bandwidth, damping ratio) or time-domain characteristics (rise time and overshoot) directly affect the controller design and tuning. The following flowchart shown in Figure 5 sums up the different strategies used for controller tuning:

The model-based approach is quite useful, especially in cases when having an actual prototype of the drone is not viable, for instance, due to budget constraints or in case someone is trying to develop his own drone model from scratch. Besides, most of the onboard drone components such as batteries or other sensors are prone to damage during testing. In such cases, having a mathematical model of the flight dynamics and control along with the external environment and sensors can help with the testing as well as precise tuning. While

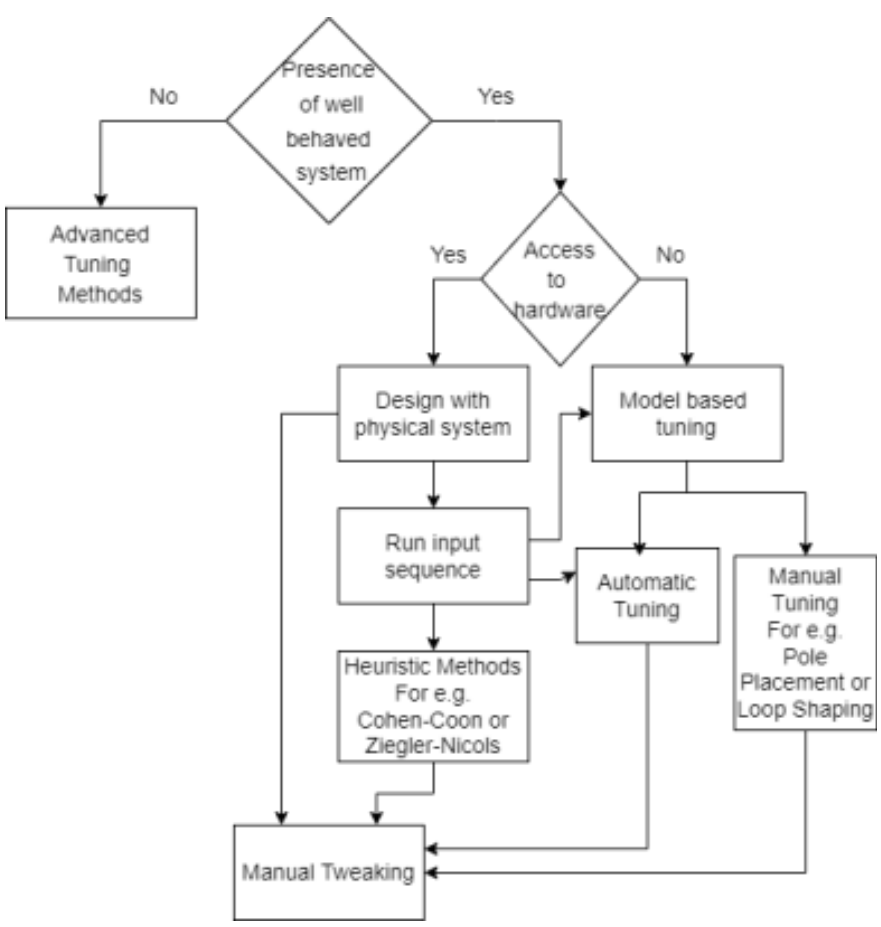

Fig. 5. PID Tuning Guide

working on this report, we have used the Simulink's Aerospace Blockset Quadrotor Project based on the Parrot mini drone for developing and testing the flight code. Figure 6 provides an architectural overview of the simulation model where the FCS module contains all the flight codes for PID control and state estimation.

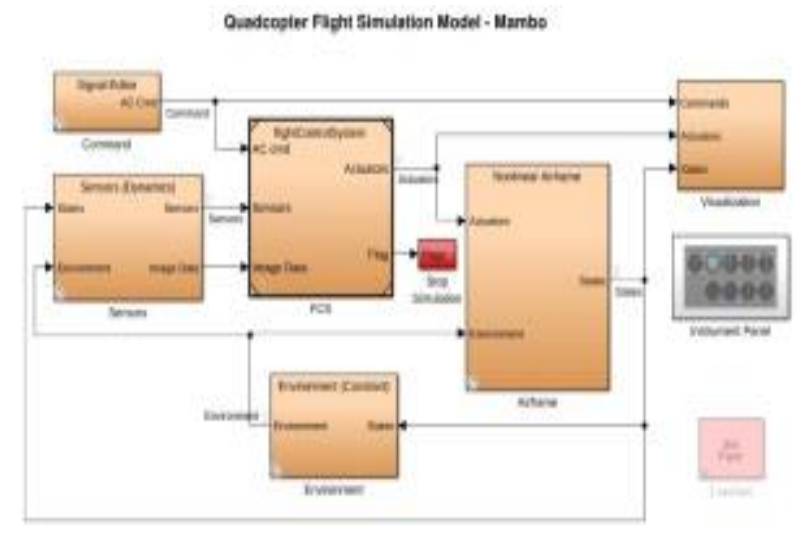

Fig. 6. Simulink Block Diagram

The rest of the modules help model the environment and provide the necessary sensor readings and simulate the drone's mechanical parameters. Using a non-linear model to simulate these parameters as close to reality and using linear analysis techniques after simplifying the flight code helps with proper controller tuning, which can then be uploaded on the drone's firmware for testing.

\section{HARDWARE ASPECTS}

Figure 7 depicts the hardware architecture of a typical autonomous quadrotor. 


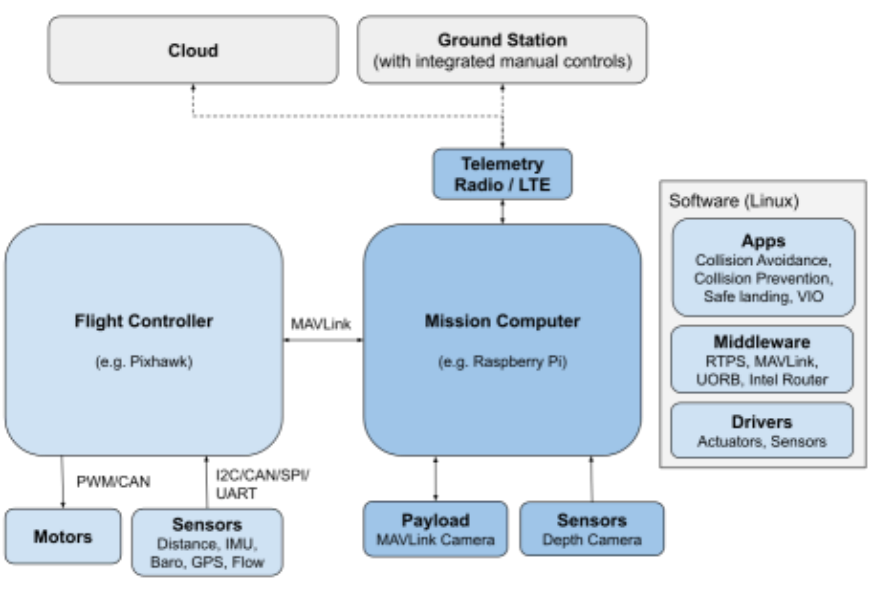

Fig. 7. PX4 System Architecture

It consists of:

- Flight Controllers:

- A circuit board that resembles the brain of the drone. Typical controllers such as the Pixhawk have 32-bit microcontroller units such as the STM32F427 Rev 3; low-level redundant sensors such as multiple IMUs, barometer integrated on the board; external buses for I2C and CAN communication with the sensors and motors; multiple UART ports to attach peripherals such as companion computer or cameras and a number of I/O pins for interfacing batteries and other analog sensors.

- The integrated processor helps with state estimation and runs the code for flight control, thereby enabling perception in the quadrotors. After running the desired closed-loop controllers, the final actuator commands are sent to the motors through the Electronic Speed Controllers(ESCs).

- Most of the quadrotors (including autonomous models) have different STM32 processors which are categorized into 8 series. Their processing powers, along with popular models employing them, are summarized in Table 3:

TABLE III SERIES of STM32 MCU

\begin{tabular}{ccc}
\hline Processor & Speed & Flash Memory \\
\hline F1 (STM32F103CBT6) & $72 \mathrm{MHz}$ & $128 \mathrm{~KB}$ \\
F3 (STM32F303CCT6) & $72 \mathrm{MHz}$ & $256 \mathrm{~KB}$ \\
F4 (STM32F405RGT6) & $168 \mathrm{MHz}$ & $1 \mathrm{MB}$ \\
F4 (STM32F411) & $100 \mathrm{MHz}$ & $512 \mathrm{~KB}$ \\
F7 (STM32F745VG) & $216 \mathrm{MHz}$ & $1 \mathrm{MB}$ \\
F7 (STM32F722RE) & $216 \mathrm{MHz}$ & $512 \mathrm{~KB}$ \\
F7 (STM32F765) & $216 \mathrm{MHz}$ & $2 \mathrm{MB} \mathrm{H7}$ \\
(STM32H750) & $480 \mathrm{MHz}$ & $128 \mathrm{~KB}$
\end{tabular}

- $\quad$ I2C communication protocol based on packet-switched serial communication is used for attaching lower-speed peripheral ICs to these MCU chips for quick intra-board communication.

- Mission Computer/ Companion Computer:
- Autonomous UAVs involve computationally expensive tasks such as object detection and obstacle avoidance which cannot be performed by the flight controller. In such cases, companion computers are used alongside the flight controller, and the two systems communicate using the MAVLink protocol. Popular examples include Raspberry Pi, Arduinos, and NVIDIA TX1. Most of them are multicore processors capable of running AI-MLbased vision/ SLAM algorithms for mapping.

- According to the results mentioned in [4], only up to 5\% of the total power is available for onboard computation. A payload of a maximum of $\sim 25 \%$ can be allotted to the onboard electronics. Table 4 briefly summarizes this estimation according to the vehicle scale:

TABLE IV

TAXONOMY OF UAVS BASED ON VEHICLE SCALE

\begin{tabular}{|c|c|c|c|}
\hline Scale & $\begin{array}{c}\text { Dimensions (in } \\
\mathrm{cm}: \mathrm{kg} \text { ) }\end{array}$ & Power (in W) & $\begin{array}{l}\text { Onboard } \\
\text { Device }\end{array}$ \\
\hline Standard & $\sim 50:>1$ & $>100$ & Desktop \\
\hline $\begin{array}{l}\text { Micro } \\
\sim 10: \sim 0.01\end{array}$ & $\begin{aligned} \sim 25: & \sim 0.5 \\
& \sim 5\end{aligned}$ & $\begin{array}{l}\sim 50 \\
\quad \mathrm{MCU}\end{array}$ & Embedded Nano \\
\hline Pico & $\sim 2<0.001$ & $\sim 0.1$ & ULP \\
\hline
\end{tabular}

- Sensors:

- * IMUs: Inertial Measurement Unit is a low-level sensor that measures the angular rate, force, and magnetic field. A micro-electromechanical system (MEMS) based IMU is typically found in all quadrotor models and is often paired with sensor fusion software by combining data from multiple sensors to estimate the heading and orientation. Depending on the type of IMU used, i.e., a 3 or 9 axis IMU (comprising accelerometer, gyroscope, and magnetometer), the flight code processes the sensor data and estimates after applying some filter to reduce the noise. Generally, gyro ratings are processed more frequently compared to accelerometer, which contains more noise, and hence more emphasis is given it while choosing an orientation system. A brief analysis of the datasheet [5] provides a glimpse of the following major parameters that should be considered while integrating a gyroscope in our system:

- Full-Scale reading, i.e., the maximum rate that the sensor can output. Usually, three scales of 250, 500, and 2000 degrees per second (DPS) are provided. Choosing a more significant scale to result in a decrease in the sensitivity of the sensor reading.

- Variation in the sensitivity with temperature. For a 3-axis L3GD20 gyro, the sensitivity might vary by $+/-2 \%$ in a temperature range of -40 to 85 degrees celsius.

- Digital zero-rate level, also called the Offset calibration error, indicates the deviation of an actual output signal from the ideal output signal 
in the absence of any acceleration. This parameter is crucial since a large value might force the system to interpret that the vehicle is moving and can interfere with the control logic. The following table 5 compiles the zero offset error for a few popular low-cost (costing around Rs.1k) gyro models:

TABLE V

COMPARISON OF ZERO OFFSET ERROR IN GYROSCOPES

\begin{tabular}{|c|c|c|}
\hline Model & $+/-250$ dps & $++/-2000 \quad d p s$ \\
\hline L3GD20 & $10 \mathrm{dps}$ & $75 \mathrm{dps}$ \\
\hline FXAS21002C & $0.3906 \mathrm{dps}$ & $0.78125 \mathrm{dps}$ \\
\hline LSM9DS0 & $10 \mathrm{dps}$ & $15 \mathrm{dps} \quad 25 \mathrm{dps}$ LSM9DS1 \\
\hline$\sim 30 \mathrm{dps}$ & $\sim 30 \mathrm{dps}$ & $\sim 30$ dps MPU-9250 \\
\hline $\begin{array}{l}\mathrm{dps} \\
\text { BMI055 }\end{array}$ & N.A. & $\begin{array}{l}\text { N.A. } \\
\text { N.A. }\end{array}$ \\
\hline
\end{tabular}

- $\quad-*$. Non-linearity: The less linear a sensor is, the less accurate the linear model of the sensor will be, and developing a controller based on inconsistent linear analysis tools can affect the drone's performance.

- Rate noise density: Measurement of the unpredictability of the system. Different manufacturers employ different techniques such as Fast Fourier Transform, Power Spectral Density or Angle Random Walk, etc., and the method used can be determined by comparing the unit of the noise density. In general, lower noise density is preferred.

- Digital Output Data Rate: The number of gyro samples per second that one can read. It is usually provided at multiple rates. In general, the higher the data rate, the faster will be the sampling of input signals that can be sensed and responded. However, higher data rates bring associated noise into the system.

* As described in the earlier sections, the sensor readings of the IMUs and other low-level sensors and estimates from the model are fused for precise state estimation. However, they are typically used only for absolute positioning and extra sensors such as GPS \& Compass, Tachometers, Distance sensors, Pressure sensors, Airspeed sensors, Optical flow, and visionbased cameras mounted on a gimbal are frequently used to enable full autonomy of the quadrotor.

* ESCs \&Motors:

- Quadrotors typically employ brushless motors driven by the flight controller using Electronic Speed Controllers (ESCs). These devices communicate using periodic pulses ranging from 1000us to 2000us and use PWM (Pulse Width Modulating) techniques for ensuring low latency. Primary buses such as UAVCANs also ensure higher update rates up to $200 \mathrm{~Hz}$ are maintained, and the motors can be controlled in real-time.

A deep dive into the real-time aspects of UAV-based embedded systems highlights the need for low-latency sensor acquisition, control responses, and robust interfaces for data
* Most of the autonomous quadrotors are equipped with a remote ground-control station for monitoring the performance of the vehicle and also to update the planners as and when required. These control stations are also used for off-board processing of a few computationally expensive algorithms such as laser or vision-based localization and mapping algorithms to reduce the load on the onboard processor. Telemetry radios are optionally used to provide a wireless connection using the MAVLink protocol. The 3DR radio manufactured by SiKRadio is a commonly used pair of transmitter-receiver hardware operating in the range of $433 \mathrm{MHz}$ to $915 \mathrm{Mhz}$.

\section{SOFTWARE ARCHITECTURE}

transfer such as I2C, SPI, CAN modes of communication. Figure 8 denotes the latency requirement at the different stages of quadrotor architecture, where the outer loop has decent timing requirements as compared to the inner loops demanding latency in microseconds.

The 32 bit STM processors used in the flight controllers lack the support for proprietary operating systems such as Linux, which is popular in the embedded systems community. Instead, these MCUs run on Real-Time Operating Systems (RTOSs), which adopt appropriate task scheduling, memory, and power management in real-time applications to meet the timing constraints. By assigning numeric priorities to the threads, which is a flow of execution through the process code at the OS level, an RTOS brings out the best from multithreaded parallel programming approaches by ensuring exclusive access to the shared data to a prioritized task, thereby avoiding concurrency errors.

The software stack of commercially available flight controllers, such as PX4, for instance, is designed, taking these requirements into account. Most of the PX4 flight controller boards, such as Pixhawk, use NuttX RTOS, which runs the uORB middleware and is used for asynchronous inter-thread communication. The publishsubscribe architecture ensures the efficiency of high-rate topics such as the $1 \mathrm{kHz}$ accelerometer updates, thereby satisfying the controller requirements.

The Robot Operating System (ROS) is another such middleware suite commonly used in robotics applications. It can easily reduce the latency at the firmware level, which is required to handle hefty sensors in autonomous systems such as LiDARs or Depth cameras and their computationally 


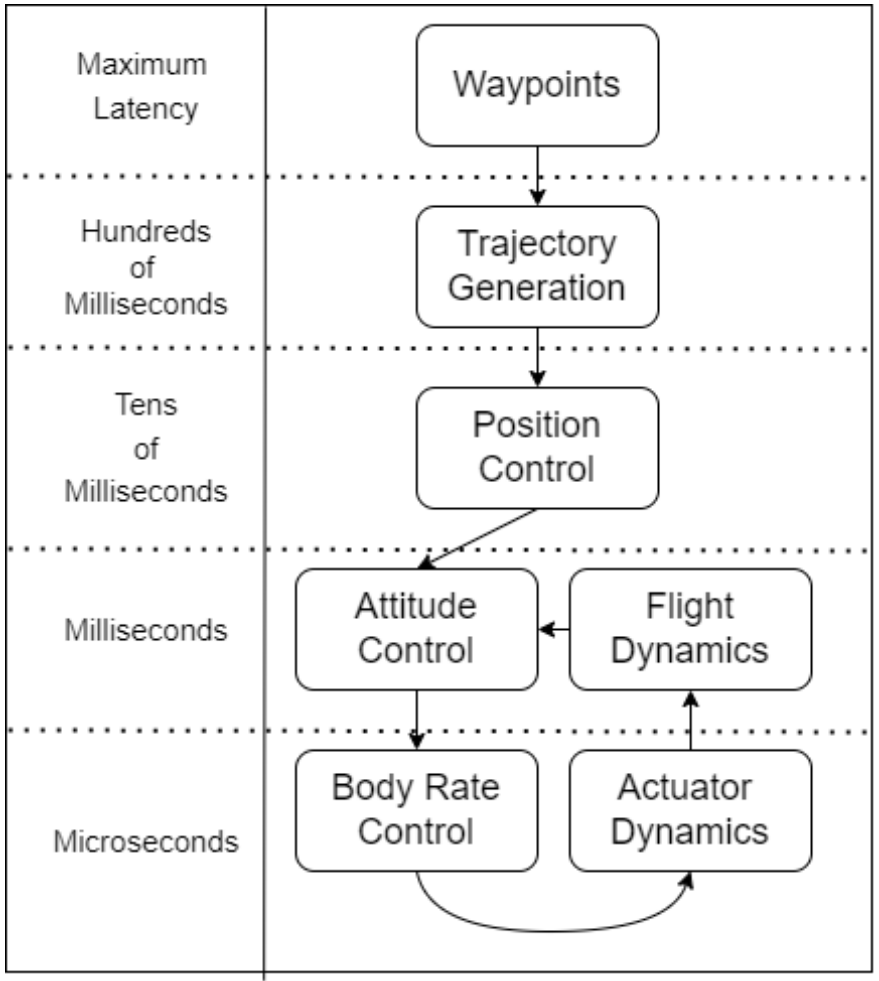

Fig. 8. Latency requirement of each stage

expensive algorithms. A lot of integrated embedded systems making use of this two middleware can be found in the academic literature where an architecture similar to the one depicted in Figure 9 is used.

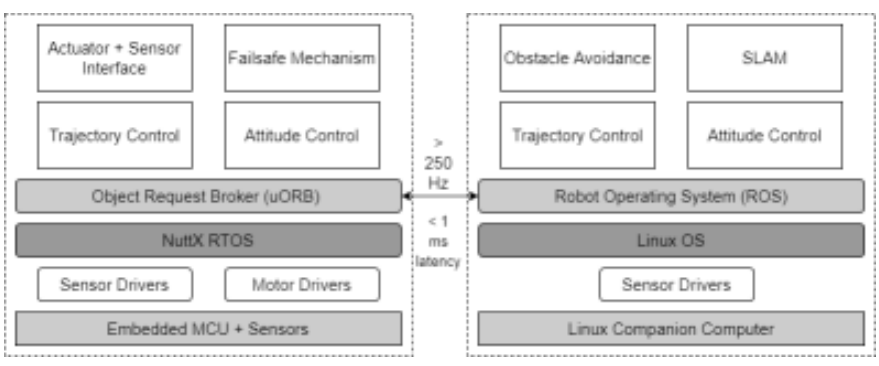

Fig. 9. Flight Controller + Companion Computer Architecture

With the introduction of ROS2 and micro-ROS [6], it is now possible to use the capabilities of ROS2 to bridge the gap between resource-constrained microcontrollers and larger processors in robotic applications. This micro-ROS framework has now allowed for easier integration of multiple MCUs chips and is being widely incorporated in both popular flight controllers and drone development platforms such as Bitcraze CrazyFlie.

\section{A BRIEF CASE STUDY OF NANO-QUADROTOR BASED DEVELOPMENT ENVIRONMENTS}

Nano-drones, having only a few tens of centimeters of diameters and a few tens of grams of weight, have proven to be extremely versatile, acting as nodes in a smart wireless network. Numerous applications of these UAVs are emerging each day where a swarm network of these quads is used to perform a task in a collaborative manner, such as recreational lighting or solving the gas source localization problem [7]. Platforms such as the Bitcraze Crazyflie 2.1 and Walkera QR Ladybug are being increasingly used for further research in this domain. Their low form-factor, however, does pose a problem for multiple MCU integration, and they often rely on external localization methods such as the Loco Positioning System, making use of optical flow or the Ultra Wide Band transmitter-receiver pairs to find the absolute 3D location of the drone. Despite its low payload carrying capacity, researchers from ETH Zurich [8] were able to successfully integrate a GAP8 System on Chip (SoC) based on the RISC-V architecture and a ULP camera on the CrazyFlie model, which was then able to execute a closed-loop, end-to-end visual pipeline based on deep Convolutional Neural Networks(CNNs) for autonomous navigation without the need to use off-board computing and sensing capabilities. The developed model was able to maneuver at a maximum translational speed of $1.5 \mathrm{~m} / \mathrm{s}$ with the algorithm running at a frequency of up to $18 \mathrm{~Hz}$ due to the optimizations made in the algorithm and at an architectural level. Integration of the MCU units used could've resulted in a further reduction in the form factor. This has been the main motivation for our work to develop a custom quadrotor model by focusing on all the important stages mentioned in the previous sections.

\section{CONCLUSION}

With a recent surge in the interest of hobbyists and academic, commercial communities to work in the field of autonomous drones, it was crucial to provide a comprehensive compilation of the underlying literature and the prerequisite knowledge necessary to work in this domain. In this report, we have provided an introduction to the modeling, estimation, and control problem for quadrotors. Sufficient information about the flight dynamics and control strategies is offered. The hardware architecture of both manual and autonomous quadrotors was studied with a short guide on the parameters affecting the design choice. The underlying system was also analyzed at a software level, followed by a brief discussion on recent advancements in the field. We hope this report serves as an introductory guide to the field of automation and aerial robotics by providing a direction for future research.

\section{ACKNOWLEDGMENT}

This report is a part of the PHN-319: Technical Communication coursework in the Department of Physics, Indian Institute of Technology Roorkee. I would like to acknowledge and thank Prof. Mayank Goswami and Mr. Ankur Kumar for allowing me to work on this topic and guiding me throughout this research work.

\section{REFERENCES}

[1] W. Giernacki et al., "Crazyflie 2.0 quadrotor as a platform for research and education in robotics and control engineering," in International Conference on Methods and Models in Automation and Robotics (MMAR). [Online]. Available: https://ieeexplore.ieee.org/document/8046794

[2] PX4 Documentation: https://docs.px4.io/master/en/

[3] V. Kumar et al., "Multirotor Aerial Vehicles: Modeling, Estimation, and Control of " Quadrotor.'[Online]. Available:https://ieeexplore.ieee.org/document/6289431

[4] R. J. Wood et al., "Progress on "Pico" Air Vehicles," in Robotics Research: The 15th International Symposium ISRR. 
[5] MEMS motion sensor: three-axis digital output gyroscope: Datasheet production data." [Online]. Available: https://pololu.com/file/0J563/L3GD20.pdf

[6] micro-ROS: ROS2 for microcontrollers. [Online]. Available: https://micro.ros.org

[7] B. Duisterhof et al., "Sniffy Bug: A Fully Autonomous Swarm of GasSeeking Nano Quadcopters in Cluttered Environments." [Online]. Available: https://arxiv.org/abs/2107.05490

[8] D. Palossi et al., "An Open Source and Open Hardware Deep Learningpowered Visual Navigation Engine for Autonomous Nano-UAVs." [Online]. Available: https://arxiv.org/abs/1905.04166 
